\title{
Infoviduality: exploring subjectivations and agentivities in a more-than-human world
}

\section{Cosimo Accoto ${ }^{1}$}

\begin{abstract}
When we talk about digital, synthetic and artificial technologies in emerging networked societies, as we can guess, the idea of "subjectivity" (or better, subjectification) connected to identity (who is what), to sensitivity (who perceives what), to agency (who does what) and, naturally, to accountability (who is responsible for what) is crucial. Intelligent objects, assistive bots, coded algorithms, swarm robotics, anticipatory softwares, autonomous vehicles, quantified bodies, data-driven agents, automated markets, sensorized ecologies all demand of us, therefore, to address the philosophical question of the "subject" with new perspectives. But what kind of subjectivity is emerging in a more-than-human world? The foundational idea that we explore here is that of the subject considered as an "elemental" process morphing and designing what I started to name "infoviduality".
\end{abstract}

Keywords: Digital Society. Elemental Subjectivity. Infoviduality.

1 Research Affiliate @ MIT (Boston). Previously Lecturer in Digital Analytics \& Big Data at IE University (Madrid), also collaborator to "Digital Markets" project, IMCA - USI University (Lugano). E-mail: cosimo@mit.edu. 


\section{Introduction}

When we talk about digital, synthetic and artificial technologies in emerging networked societies, as we can guess, the idea of "subjectivity" (or better, subjectification) connected to identity (who is what), to sensitivity (who perceives what), to agency (who does what) and, naturally, to accountability (who is responsible for what) is crucial. Nowadays, philosophy, technology, jurisprudence and politics - to name a few - disciplinarily contend the relevance of these dimensions, which in turn are central to the ways whereby we can create, eventually, participation and value for collectivities and citizens, developing the decentralized and distributed governance of public affairs and private business. Intelligent objects, assistive bots, coded algorithms, swarm robotics, anticipatory softwares, autonomous vehicles, quantified bodies, data-driven agents, automated markets, sensorized ecologies all demand of us, therefore, to address the philosophical question of the "subject" with new perspectives. But what kind of subjectivity is emerging in a more-than-human world? The foundational idea that we explore here is that of the subject considered as an "elemental" process morphing and designing what I started to name "infoviduality". Elemental is a term used in media studies to indicate the new dimension of presence and action introduced and orchestrated by sensory, digital and artificial networks and their dynamics operating at scales which are, at the same time, above and below the so-called "human". We must not, of course, confuse elemental with environmental understood in a "naturalistic" sense. In an elemental perspective, subjectivity must be rethought and reconceptualized as it is no longer identifiable as the privileged prerogative of individual human actors. This philosophical perspective, therefore, insists on the in-human aspects of the network dimension, orienting itself to embrace a more dispersive and distributive vision of the capacity to act (agency) within the networks of sensors and actuators as tecno-ecological instantiations of humans, vegetables, animals, things in a reticular mutual perturbation. Therefore, a fundamental rethinking of the human and non-human experience (I renamed it "ex-perience") is in order to be able to fit them adequately into an elemental thought. We must also abandon the easy dichotomies between human and non-human and begin to think of the "human" as one of the possible declinations, embodiments and enactments of elemental subjectivity. And, 
then, adopt a perspective where subjectivity is an emerging configuration of the elemental between the moving overlap of micro-scale (sensors, actuators, chips, transducers, waves, edges, perturbations) and the macroscale (networks, clouds, protocols, grids, stacks, chains, algorithms and platforms).

In so doing, we must, for a moment, withhold the idea of agency and the notion of smartness as connected and similar to the common understanding of human intelligence: computer science and moral philosophy (among many other disciplines) are not on the same wavelength when they talk about agency. We must open ourselves to considering infovidualities (in form of emergencies or instances) that act in the world, but without necessarily having the characteristics that, as a rule, we assign to human intentional action. We can now try to indicate some of the main characteristics of this new infoviduality; although they are separate characteristics, they are strongly intertwined with each other.

\section{Data-Driven Agency}

The first important aspect is that we are dealing with a kind of data-driven subjectivity (human as well as non-human) that is guided or enhanced by data, from the data-sense we mentioned at the beginning. Mark Hansen (2014) talks, in his recent book Feed Forward, about data-enhanced actors and about "datasense", a new sensing capacity. Datasense implies the production of sensitivity due to data collection operations (also in a collective participatory sensing way for citizens). To be clearer: the data create a totally new domain of sensitivity (so, the data as experience). According to Hansen, the perspective is dual: access to data is also data production. The data do not mediate (if mediate is still the right verb to use) our senses, but mediate sensation as such and in itself. Sensibility mediation is also sensitivity production.

The impact of this data-driven agency will increasingly be exploited by machine learning and its ability to model reality in a predictive advance for the future. And if a machine or a data-driven agent (actants) defines a real situation, it is also real in its consequences. So, regardless of whether or not we decide to consider real and concrete (and not just immaterial and virtuous), algorithms, bots, assistants and artificial lives, they are certainly real in the consequences of their actions. I also started to 
rename as "infoviduality" and "infovidual" this emerging data-driven agency to stress the protentive - not only retentive - dimension of computing and modeling data (biometric, socio- metric, ecometric, etc.). In my perspective, retentive means past-oriented data and protentive means future-oriented data (see fig.1). In fact, the data impact covers an agency continuum: from privacy (protection) to destiny (protention).

Figure 1 - Infoviduality dimensions and rights

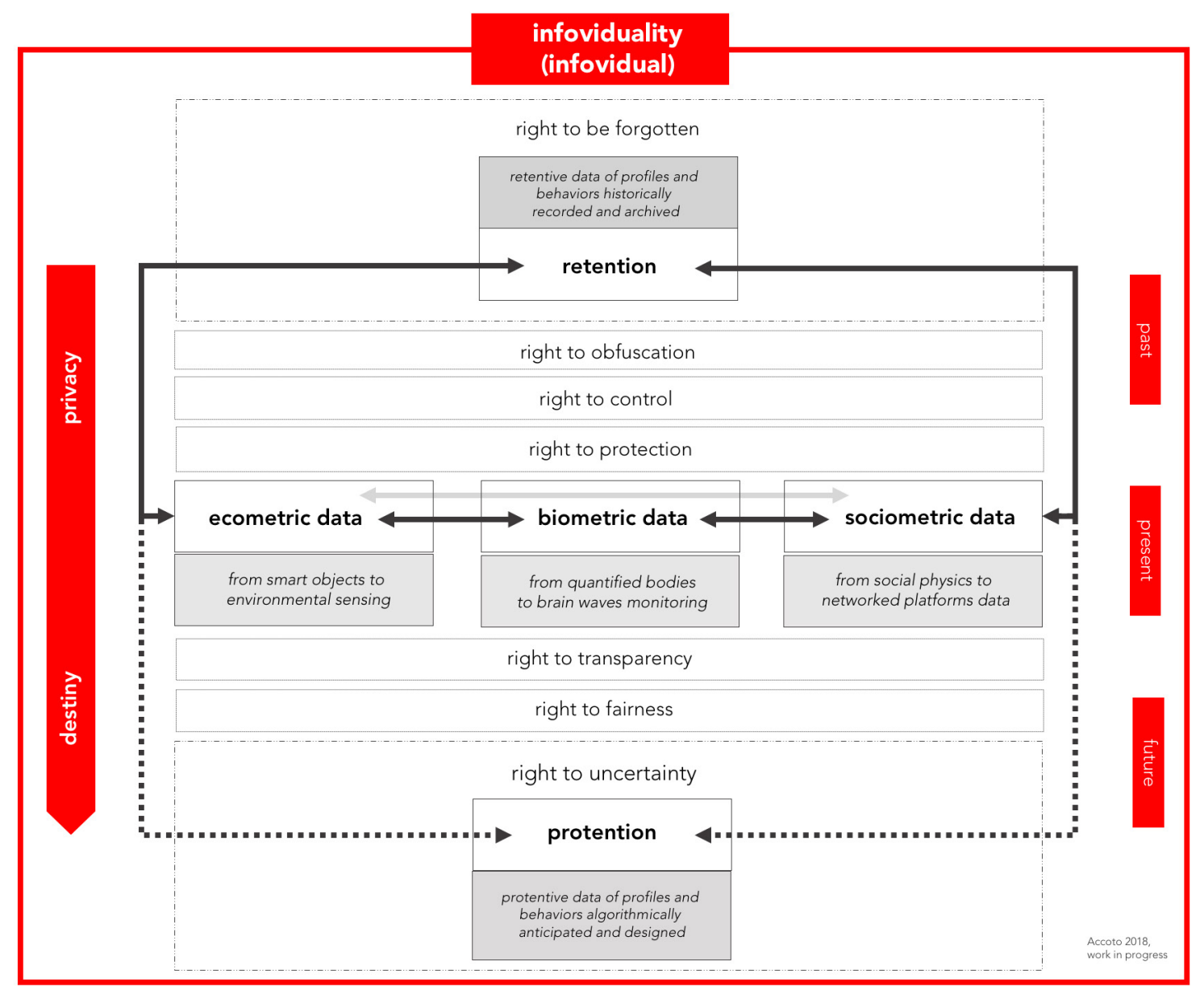

\section{Distributed Agency}

We also talk about infovidualities that are distributed. Subjectivity is disseminated between micro and macro dimensions, between scales and layers, between probability calculations and forecasting models. In fact, what is happening is that the philosophical, religious, historical and cultural systems that have given life in the past to the concept of the "individual" (and to its counterparts such as community and so- 
ciety) are eroding in favour of an emerging age of "dividuality" - as the philosopher Deleuze would say - with all its impacts both in a positive (expected empowerment) and also in a critical perspective (risk of enslavement). In the calculation of the "human" (citizen), we have moved from the age of the average individual to the age of digital dividuality and now to the age of infoviduality. For this transition from "individuality" to "dividuality" to my concept of "infoviduality", the calculation and quantification of the body and its organs, of gestures and movements, of behaviors and interactions in a more-than-human world become the means to construct the idea and the practice of a new subjectivity, multiplied, distributed and mostly anticipated. Objects, ambients, nets and bots animate tecno-ecological interactions the responsibility of which is often not easy to place and determine, spread as it is between algorithms, networks, databases and protocols (not just humans). Cultural theories are used to say that media are "extensions" of humans. Instead, I think it's time to say that, in many cases, media are and increasingly will be "abstentions" of humans.

\section{Automated Agency}

The elemental subjectivity is, moreover, more and more automated, whether it concerns human subjects or refers to non-human entities. In computational worlds, artificial agents/actants are a type of software able to act autonomously, perceiving in some way the external environment and producing a certain adaptive change. A car without a driver and a self-regulating thermostat are active, an energy network that distributes services using real-time feedback on consumption to determine prices and disbursements is also an agent. There are, of course, different levels in the ability to act as philosopher of law Hildebrandt (2015) suggests in Smart Technologies and the Ends of Law: agents driven by deterministic algorithms (perception and response are predefined), agents that employ learning algorithms (and that, in a supervised, unsupervised or reinforced way, build new knowledge), agents based on a multi-agency system (entities of the first and second order that interact even, on occasion negotiating their respective objectives in an emerging way), or completely autonomous agents (biological or artificial, able to survive outside the software architectures even if they are constituted by computational systems). 
But automation is not just related to robots or machines. I think automation is becoming a more general orientation of our society. I tend to consider automation, paradoxically, as a "new (human) institution". To me automation, to give an example, is also instantiated by the blockchain protocol: in this case, the protocol is an automated algorithmic mechanism that mobilize self-enforced interactions among nodes in a peer-to-peer network. Automation is not just a mechanism. It's more (and more) a new ecological force and less (and less) a mere machinic process.

\section{Precognitive Agency}

Finally, we are experiencing subjectivities anticipated by the feed-forward structure that we have quoted above (anticipatory action, tertiary protection, near time). If the media and mediation technologies of the 2oth century had operations based on registration, archiving and transmission, the sensing and mining technologies of the 21st century are a new architecture that enhances the new anticipated temporal dimensions). I started to frame metaphorically this paradigm change as the shift from media technology as "archive" to media technology as "oracle". We no longer live in an archival age, we now live in the oracular age. As Hildebrandt (2015, p.11) says:

Pre-emptive smart environments begin to transform our dealings with artefacts. At some points, we will become aware of the fact that we are being watched and anticipated by machines and we will try to figure out how the infrastructure "reads" us and with whom it shares its knowledge of our preferences and of the risks we incorporate.

Going from the analysis of profiling a behaviour to that of anticipating it would seem to be a brief and innocuous step, but in reality, it has deep epistemological and ontological implications. Philosophically, we must ask ourselves: is this an anticipation of the future (let's say, for example, that we would have bought such a book in the following months anyway and that the algorithm was only anticipating our action) or it is about the actual creation of that future (in fact we would never have discovered that book and would never have bought it if it had not been suggested by the platform)?

Therefore, perception and consciousness will increasingly become elemental dimensions. Artificial sensing technologies will directly mediate the causal 
infrastructure of worldly sensibility in micro-temporality and micro-sensoriality independent of consciousness, fueling our near future, influencing the action of the decentralized and distributed consciousness of the world and shaping the active role of the person (citizen) aimed at achieving their goals (feed-forward) or the goals of those who will have the practical and political opportunity to act on these dimensions. This will be further accelerated by blockchain institutional emergence. In a blockchain wold, contractual relations algorithmically predetermined, encrypted, decentralized and self-executable would replace historical, private and public organizations, regulated by traditional communication and negotiation mechanisms.

In the future, the idea is to make "human" organizations evolve toward more participative forms (voluntary affiliation), collaborative (oriented toward common objectives), cooperative (including sharing the generated value), distributed (thanks to the propagation and multiplication of nodes of extended in-human networks), decentralized (in highly scalable mode) and automated (thanks to software code, algorithms and artificial intelligences for self-sustaining and to share value in an equal way). Code and protocol are changing the game. The code is an executable writing of the world. It produces action on our life and on our reality, it has the ability to make things happen. If we think about it, this is precisely one of the founding prerogatives of future forms of sovereignty. And many questions will emerge about governmentality and agency, individual as well as collective, private and public, human and inhuman, conscious and unconscious, local and scaling up. Certainly, it is no longer just a matter of the government understood in legal, contractualistic or biopolitical terms, but more and more of the exercise of power in terms of distributed and decentralized protocols and algorithms. Quite the opposite, increasingly traditional aspects of governance of the so-called "self" and "social" are being subsumed and reabsorbed by forms of software-driven management and modulation (and control).

\section{References}

HANSEN, M.. Feed-Forward; on the future of twenty-first-century media. Chicago: University of Chicago Press, 2014.

HILDEBRANDT, M.. Smart Technologies and the End(s) of Law. Cheltenham and Camberley (UK); Northampton, Massachusetts (USA): Edward Elgar, 2015. 